\title{
Feasibility and accuracy of second-look laparoscopy after gastrectomy for gastric cancer: reply to letter to the editor
}

\author{
Kentaro Inoue $\cdot$ Yasushi Nakane $\cdot$ A-Hon Kwon
}

Published online: 12 March 2010

(C) Springer Science+Business Media, LLC 2010

Our primary purpose for performing second-look laparoscopy [1] is to identify patients without clinical evidence of disease who nevertheless harbor microscopic disease and likely would benefit from further therapy. Early identification of peritoneal progression is helpful because it allows patients to receive further treatment while they have good organ function and performance status. Another purpose of second-look laparoscopy is to identify patients who have had a complete pathologic response to chemotherapy. We consider that these patients are likely to be cured and thus could discontinue chemotherapy.

We agree with Zoras et al. [2] that robust and early assessment of the treatment response is crucial because of the very high costs and long-term duration of the combination of the systemic chemotherapy regimen and targeted therapy. Second-look laparoscopy could lead to the rational decision to stop treatment in patients with peritoneal progression during expensive multimodal therapy.

Currently, laparoscopy is the only method that allows accurate diagnosis of peritoneal dissemination from gastric cancer. Advances in laparoscopic techniques and instruments have been remarkable, with a steady increase in advanced endoscopic surgery, e.g., laparoscopic gastrectomy and colectomy. The development of new products such as adhesion barriers is also progressing. Various active chemotherapy regimens with targeted drugs could soon be available. We believe that second-look laparoscopy or even laparoscopic follow-up could be feasible and make an important contribution to improving the survival and quality of life of patients with gastric cancer.

Disclosure Drs. Inoue, Nakane, and Kwon have no conflicts of interest or financial ties to disclose.

\section{References}

1. Inoue K, Nakane Y, Michiura T, Yamaki S, Yui R, Sakuramoto K, Iwai A, Tokuhara K, Araki Y, Kim S, Nakai K, Sato M, Yamamichi K, Kwon A (2009) Feasibility and accuracy of secondlook laparoscopy after gastrectomy for gastric cancer. Surg Endosc 23:2307-2313

2. Zoras O et al (2010) Feasibility and accuracy of second-look laparoscopy after gastrectomy for gastric cancer [letter to the editor]. Surg Endosc 24
K. Inoue $(\varangle) \cdot$ Y. Nakane $\cdot$ A.-H. Kwon

Department of Surgery, Kansai Medical University,

Hirakata, Osaka, Japan

e-mail: inoueke@hirakata.kmu.ac.jp 